Methods: medical and pedagogical, observation, examination, assessment.

Results: Variants of the child's psychophysical development: Option 1. (75\%): children with a predominance of violations in behavior, emotional and volitional sphere. There is aggressiveness, inconsistency and impulsiveness of actions, lack of distance with an adult, and difficulties in complying with accepted norms and rules. Option 2 (25\%): children with the following manifestations: timidity, tearfulness, distrust, fears, lack of initiative. All children have difficulty sleeping, eating disorders, and frequent psychosomatic illnesses.

Conclusions: Children with intellectual disabilities in an inclusive practice need comprehensive assistance, taking into account different variants of their psychophysical characteristics. The studied children were found to have neurotic and neurosis-like disorders, as well as pathological personality development. All children have: low performance, lability of the nervous system, lack of voluntary regulation, impaired activity, learning difficulties. There are behavioral and mental disorders that require medical, psychological and pedagogical rehabilitation.

Keywords: children with intellectual disabilities; inclusive education; learning difficulties; behavior disorder

\section{Mental health care}

\section{EPP0715}

The effect of exercise on the cognitive and physical function of patients with dementia

\author{
V. Papatsimpas ${ }^{1 \star}$, S. Vrouva ${ }^{2}$ and D. Bakalidou ${ }^{3}$ \\ ${ }^{1}$ Physiotherapy Department, General Hospital Athens "G. \\ Gennimatas”, Athens, Greece; ${ }^{2}$ Physiotherapy Department, \\ 401 General Military Hospital of Athens, Athens, Greece and \\ ${ }^{3}$ Department Physiotherapy, University of West Attica, Egaleo, Greece \\ ${ }^{\star}$ Corresponding author. \\ doi: 10.1192/j.eurpsy.2021.1034
}

Introduction: Dementia is characterized by a decrease in mental functions, while disorders of balance, coordination of movements and gait are gradually added. In recent years there has been a growing interest in the role of exercise as a therapeutic strategy for people with dementia.

Objectives: The aim of this study was to investigate the effect of different types of exercise and its parameters on cognitive and physical function in patients with dementia after reviewing the relevant literature.

Methods: Review of the literature based on the research of original scientific articles published in the electronic databases PubMed / Medline and Google scholar using as keywords the terms dementia, cognitive function, physical function, functionality, aerobic exercise, resistance exercise.

Results: A review in the literature highlights the beneficial effect of exercise on patients with dementia. Aerobic exercise and mixed interventions have been studied more, while resistance interventions have been less studied. All three types of exercise have shown positive effects. The methodology differences of the studies make it difficult to draw definitive conclusions about the optimal intervention in the cognitive and physical function for the optimal result, the type of exercise, the duration, the frequency and the intensity.
Conclusions: Exercise (physical) may help maintain or improve cognitive function and functionality in patients with dementia but additional study is needed to clarify optimal intervention and establish guidelines.

Keywords: dementia; Exercise; cognitive function; physical function

\section{EPP0716}

Burden of the family caregivers of the person with mental illness in portugal: A cross sectional study

C. Laranjeira* and A. Querido

Citechcare, Polytechnic of Leiria, Leiria, Portugal

${ }^{*}$ Corresponding author.

doi: 10.1192/j.eurpsy.2021.1035

Introduction: The provision of mental health care should be promoted at the community level, in order to facilitate their recovery process. Thus, the people who care for these individuals go through a situational transition, as they have to play the role of Family Caregivers (FC).

Objectives: a) to characterize the FC burden of the person with Mental Illness; and b) to correlate FC overload with the variables age, gender, cohabitation, degree of kinship and level of education. Methods: A cross-sectional correlational study was conducted. The sample consisted of FC who went to consultations and to the inpatient psychiatric unit of a portuguese hospital. Data were collected through a questionnaire which included sociodemographic data, the Lawton-Brody Index and the Zarit Burden Interview (ZBI). Results: Of the 119 FC, $66.4 \%$ were female, with an average age of 53.8 years. $73.1 \%$ of FC cohabited with the person they care for, in which the majority was cared for by their child or spouse, $45.2 \%$ and $36.1 \%$, respectively. It should also be noted that $71.4 \%$ felt the need for more support from health professionals. As for autonomy, 52\% of the individuals cared for by FC were moderately dependent. It was verified that about $45 \%$ of the FC had an intense overload. Female gender has greater overload and that there are no significant differences between the level of education and cohabitation.

Conclusions: Considering the results, it is understood the importance of valuing FC, as a target and care partners, in order to reduce the burden, they feel when caring for people with MI.

Keywords: Caregiver Exaustion; Family Caregivers; Person with Mental Illness; Situational Transition

\section{EPP0717}

Profile of mood states-27: A valid and reliable measure of negative and positive affect for brazilian pregnant women
M. Barros ${ }^{1,2 *}$, M. Aguiar $^{3}$, A. Macedo ${ }^{4}$ and A.T. Pereira ${ }^{5}$
${ }^{1}$ Departamento De Ciências Naturais, Universidade do Sudoeste da Bahia - UESB, Vitória da Conquista, Bahia, Brazil; ${ }^{2}$ Instituto De Psicologia Médica, Universidade de Coimbra, Coimbra, Portugal; ${ }^{3}$ Pos Graduação Em Psicologia Da Saúde, Universidade Federal da Bahia, Vitória da Conquista, Brazil; ${ }^{4}$ Institute Of Psychological Medicine, Faculty Of Medicine, University of Coimbra, Coimbra, Portugal and ${ }^{5}$ Institute Of Psychological Medicine, Faculty Of Medicine, University of Coimbra, coimbra, Portugal 
${ }^{\star}$ Corresponding author.

doi: 10.1192/j.eurpsy.2021.1036

Introduction: The original Profile of Mood States (McNair et al. 1971 ) is composed of 65 items to measure six affect dimensions. There is a Portuguese shorter version (POMS-27) for the perinatal period (Bos et al. 2013), which reduced the number of items (adjectives) down to 27. Although briefer, it still covers relevant mood states with good psychometric properties, being considered a good alternative to use during this demanding and busy period.

Objectives: To test if PoMS-27, composed of four dimensions (Depression, Anxiety, Vigour, Fatigue) and one second-order factor (Negative Affect, composed by the former two) is valid and reliable to be used with pregnant women from Brazil.

Methods: After confirming the items semantic equivalence and slightly adapt two adjectives from European to Brazilian Portuguese, 350 pregnant women (Mean age: $30.01 \pm 5.452$; Mean gestation weeks $=25.17 \pm 6.55$ ), with uncomplicated pregnancies, completed the PoMS-27 and the Brazilian recently validated versions of Perinatal Depression Screening Scale and Perinatal Anxiety Screening Scale (PDSS; PASS; Barros et al. 2021). SPSS and AMOS software were used.

Results: After deleting two items (20 and 22) and some errors were correlated, CFA indicated that the PoMS-25 four-dimensional model presented an acceptable fit $(\chi 2=3.163$; $\mathrm{RMSEA}=.079$, $\mathrm{CFI}=.868, \mathrm{TLI}=.849)$. The Cronbach alphas of the dimensions were: Depression (.82), Anxiety (.86), Negative Affect (.89), Fatigue (.80) and Vigor (.50).

Conclusions: Within this psychometric study we found useful, valid and reliable measure of negative and positive affect for Brazilian pregnant women. From now on we will denominate it Profile of Mood States-25Br

Keywords: negative affect; Depression; validation; pregnancy

\section{EPP0718}

\section{Brazilian version of the postpartum depression screening scale-24}

\author{
M. Barros ${ }^{1,2 *}$, M. Aguiar $^{3}$, A. Macedo ${ }^{4}$ and A.T. Pereira ${ }^{5}$
}

${ }^{1}$ Departamento De Ciências Naturais, Universidade do Sudoeste da Bahia - UESB, Vitória da Conquista, Bahia, Brazil; ${ }^{2}$ Instituto De Psicologia Médica, Universidade de Coimbra, Coimbra, Portugal; ${ }^{3}$ Pos Graduação Em Psicologia Da Saúde, Universidade Federal da Bahia, Vitória da Conquista, Brazil; ${ }^{4}$ Institute Of Psychological Medicine, Faculty Of Medicine, University of Coimbra, Coimbra, Portugal and ${ }^{5}$ Institute Of Psychological Medicine, Faculty Of Medicine, University of Coimbra, coimbra, Portugal

${ }^{*}$ Corresponding author.

doi: 10.1192/j.eurpsy.2021.1037

Introduction: The PDSS-24 is a Portuguese short version of the Postpartum Depression Screening Scale (Beck and Gable, 2002). Items were selected on the basis of exploratory factor analysis (those with loadings $>.60$ ). The PDSS-24 proved to be superior to the 35 -items PDSS in reliability, validity and screening ability (Pereira et al. 2013).

Objectives: To analyze the psychometric properties (construct validity using Confirmatory Factor Analysis, discriminant validity and reliability) of the Brazilian preliminary version of PDSS-24
Methods: After confirming the items semantic equivalence and slightly adapt two adjectives from European to Brazilian Portuguese, 350 pregnant women (Mean age: $30.01 \pm 5.452$; Mean gestation weeks $=25.17 \pm 6.55$ ), with uncomplicated pregnancies, completed the PDSS-24 and the Brazilian recently validated versions of Profile of Mood States-25 (PoMS; Barros et al. 2021). SPSS and AMOS software were used.

Results: After some errors were correlated the multidimensional second-order model of PDSS-24 presented an aceptable fit $(\chi 2=3.448$; $\mathrm{RMSEA}=.099 ; \mathrm{CFI}=.817$, TLI $=.886, \mathrm{GFI}=.886)$. The PDSS Cronbach's alpha for the total was $\alpha=.90$. Cronbach alpha was .90 for the total and $>.75$ for the dimensions. Appling the Portuguese validated cut-off score for Major Depression/DSM-5 $(>42)$ to this sample 224 (64.0\%) participants presented clinical relevant depressive symptoms.

Conclusions: The Brazilian PDSS-24 has acceptable validity and reliability. The percentage of women with high depressive symptomatology is three times higher than the figures reported in Portuguese Studies. This can be partly explained by the fact that data collection was done during the COVID19 pandemic. It is important to determine the PDSS cut-offs to screen for perinatal depression in Brazil.

Keywords: Reliability; Depression; Validity; Scale

\section{EPP0722}

History of medical body: Demystifying the continuum of psychiatry and psychoanalysis

\section{Gairola*}

School Of Human Studies, Ambedkar University Delhi, Delhi, India ${ }^{*}$ Corresponding author.

doi: 10.1192/j.eurpsy.2021.1038

Introduction: The aim of this paper lies in demystifying, historicizing, and de-alienating the relationship between psychiatry and psychoanalysis. Both psychiatry and psychoanalysis inform each other and are informed by each other in various ways which are on one hand similar and on the other hand unique. Medicine can be seen psychoanalytically, and a presenting complaint to a psychoanalytic psychotherapist can be seen in psychiatric terms.

Objectives: This paper theorizes the 'and' between psychiatry and psychoanalysis. What is that bridging telling us? There is already an invisible 'and' which joins psychiatry and psychoanalysis even before this visible 'and' was placed in between them. The effort here is not to undermine the difference. It is to be aware that the thing which separates is also a thing which connects. In other words, each separation is a link. It is to understand how psychiatrists have contributed to the method and practice of psychoanalysis and visa versa. It is about witnessing the continuum that is everpresent between psychiatry, psychology, and psychoanalysis.

Methods: This research used primary sources like books and articles to historicize the psychiatric conceptualization of the medical body. Results: It shows how psychiatry and psychoanalysis inform each other and are informed by each other. To locate the historical conceptions which are still ever-present in modern psychiatry. How the 18th-century division between normal and abnormal is based on an older division between good and evil.

Conclusions: Good and evil continue to operate in the realm of psychiatry and the confessional becomes a prime source for psychiatry and psychoanalysis. 